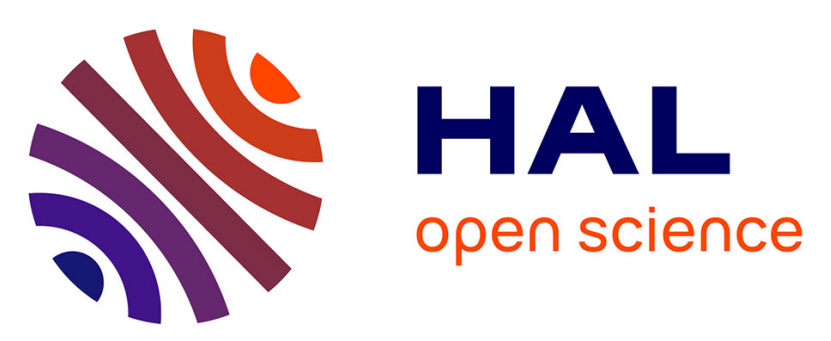

\title{
Does motion affect liver stiffness estimates in shear wave elastography? Phantom and clinical study
} \author{
Claire Pellot-Barakat, Linda Chami, Jean Michel Correas, Muriel Lefort,
} Olivier Lucidarme

\section{- To cite this version:}

Claire Pellot-Barakat, Linda Chami, Jean Michel Correas, Muriel Lefort, Olivier Lucidarme. Does motion affect liver stiffness estimates in shear wave elastography? Phantom and clinical study. European Journal of Radiology, 2016, 85 (9), pp.1645 - 1650. 10.1016/j.ejrad.2016.07.001 . hal-01436283

\section{HAL Id: hal-01436283 \\ https://hal.sorbonne-universite.fr/hal-01436283}

Submitted on 16 Jan 2017

HAL is a multi-disciplinary open access archive for the deposit and dissemination of scientific research documents, whether they are published or not. The documents may come from teaching and research institutions in France or abroad, or from public or private research centers.
L'archive ouverte pluridisciplinaire HAL, est destinée au dépôt et à la diffusion de documents scientifiques de niveau recherche, publiés ou non, émanant des établissements d'enseignement et de recherche français ou étrangers, des laboratoires publics ou privés. 


\section{Does motion affect liver stiffness estimates in Shear Wave Elastography?}

\section{Phantom and clinical study}

Claire Pellot-Barakat ${ }^{1}$, Linda Chami ${ }^{2-4}$, Jean Michel Correas ${ }^{3}$, Muriel Lefort ${ }^{4}$, Olivier Lucidarme $e^{2-4}$

\section{Author Names and e-mail:}

Claire Pellot-Barakat, claire.barakat@inserm.fr

Linda Chami, linda.chami@yahoo.com

Muriel Lefort, Muriel.Lefort@lib.upmc.fr,

Jean Michel Correas, jean-michel.correas@nck.aphp.fr

Olivier Lucidarme, olivier.lucidarme@psl.aphp.fr

\section{Institutional affiliations:}

${ }^{1}$ Laboratoire Imagerie Moléculaire In Vivo (IMIV), UMR 1023 Inserm/CEA/Université Paris Sud - ERL 9218 CNRS, CEA/I2BM/SHFJ Orsay, France

${ }^{2}$ Radiology department, Hôpital Pitié-Salpétrière, APHP, Paris, France

${ }^{3}$ Radiology department, Hôpital Necker, APHP, Paris, France

${ }^{4}$ Sorbonne Universités, UPMC Univ Paris 06, CNRS, INSERM, Laboratoire d'Imagerie Biomédicale (LIB), 75006 (ou 75013), Paris, France

\section{Corresponding author}

\section{Pr Olivier Lucidarme}

Service de radiologie centrale

Groupe hospitalier Pitié Salpêtrière

47-83 Bd de l'hôpital

75013 Paris

France

phone: 33142176322

fax: 33142178224

olivier.lucidarme@psl.aphp.fr 


\title{
Does motion affect liver stiffness estimates in Shear Wave Elastography? \\ Phantom and clinical study
}

\begin{abstract}
This study was undertaken to evaluate the impact of free-breathing (FB) vs. Apnea on Shearwave elastography (SWE) measurements.

Quantitative liver-stiffness measurements were obtained during FB and Apnea for 97 patients with various body-morphologies and liver textures. Quality indexes of FB and Apnea elasticity maps (percentage of non-filling (PNF), temporal (TV) and spatial (SV) variabilities) were computed. SWE measurements were also obtained from an homogeneous phantom at rest and during a mechanically-induced motion.

Liver-stiffness values estimated from FB and Apnea acquisitions were correlated, particularly for homogeneous livers $(r=0.76, P<0.001)$ and favorable body-morphologies $(r=0.68$, $P<0.001)$. However FB values were consistently $20-25 \%$ lower than Apnea ones $(P<0.001)$. FB also systematically resulted in degradation of TV $(P<0.005)$ and PNF $(P<0.001)$ compared to Apnea but had no impact on SV. With the phantom, no differences between SWE measurements at rest and during motion were observed.

Apnea and FB measurements are highly correlated, although FB data quality is degraded compared to Apnea and estimated stiffness in FB is systematically lower than in Apnea. These discrepancies between rest and motion states were observed for patients but not for phantom data, suggesting that patient breath-holding impacts liver stiffness.
\end{abstract}

\section{Introduction}

Staging hepatic fibrosis is crucial for the diagnosis of chronic liver disease and therapeutic decision-making. Recent technological advances have enabled liver-fibrosis staging using noninvasive ultrasound techniques, such as elastography, which provides an estimation of liver 
stiffness [1,2]. Conventional ultrasound-elastography techniques or strain imaging evaluate how tissues respond to a static compressive force and provide a qualitative map of elasticity [3]. Unlike strain imaging, shear-wave elastography (SWE) generates a low-frequency shear wave with the acoustic force of the ultrasound beam to push the tissue. The ultrasound probe then captures the shear wave propagation and measures its velocity. The result enables noninvasive, quantitative assessment of tissue elasticity (in kiloPascals, $\mathrm{kPa}$ ), which is related to shear-wave velocity.

SWE usefulness was demonstrated for the liver, where tissue elasticity correlates with fibrosis severity [4]. Hence, SWE is now considered a useful tool for staging liver fibrosis noninvasively. Most liver-stiffness measurements reported in the literature were obtained using a breath-hold technique to minimize potential artifacts and uncertainties due to breathing movements during the measurement of the shear-wave velocity [5-7]. That context was totally justified in early elastography studies based on strain imaging, because accurate estimation of the displacement field induced by the compressive force required the absence of any respiratory movement between the pre- and post-compressive states. However, according to the physical principles of SWE, which provides up to 10,000 frames/s to follow the real-time propagation of the pulsed shear waves in the body [8], we could theoretically question the need for apnea when measuring liver stiffness. Notably, breathing movements are much slower than the shearwave-propagation velocity and respiratory frequency is much lower than the image-acquisition rate required to assess the shear-wave velocity. Moreover, for numerous patients, it is difficult to achieve apnea.

In the current study, we sought to evaluate whether liver movements, induced by free breathing during SWE acquisition, influence liver stiffness measurements and, if so, to understand how and why motion impacts SWE measurements. To our knowledge, the feasibility of FB and its 
influence on SWE measurements of liver stiffness in clinical practice have not been previously reported.

\section{Materials and Methods}

Clinical Study: Patients. This study was approved by the local research Ethics Committee. Consecutive patients $(n=109)$ referred for abdominal ultrasound examination for various reasons, including chronic liver disease, hepatitis, liver damage after chemotherapy, liver transplant follow-up, characterization of focal lesions or abdominal pain, gallstones, etc. were included from October 2013 to April 2014 after informed consent was obtained (the Ethics Committee waived written consent).

Acquisition Protocol. All patients underwent conventional grayscale ultrasonography, during which SWE measurements of the right liver were obtained with an Aixplorer ${ }^{\mathrm{TM}}$ (SuperSonic Imagine, Aix-en-Provence, France) with the abdominal curved transducer (SC6-1) always using the same liver-scanning presettings. Patients were told to fast prior to the examination. Patients were placed in the supine position with the right arm in abduction and the transducer was placed in the intercostal space to examine the right lobe of the liver. Abdominal ultrasound imaging was performed by two experienced radiologists. Acquisitions were obtained using the SWE-imaging mode with 'medium persistence' and 'penetration' settings. Two 10-second SWE video clips were acquired for each patient, always in the same order: the first was obtained while the patient lay quietly in the supine position on the examination table in FB mode, with no instructions given to the patient, such as 'breath freely' or 'do not hold your breath', to prevent possible exaggerated respiration; the second was recorded in apnea mode. As in routine practice, apnea was obtained by asking the patient to stop breathing while in quiet respiration, 
without prior lung filling. For most patients, apnea thus occurred while in intermediate breathing, which is recommended by the ESFSUMB [9].

Data Collection: SWE measurements. The SWE video clips were retrospectively analyzed by an engineer blinded to the pathological liver conditions with the AAE (for automatic analysis of elasticity maps) software [10]. SWE measurements were made in the frame considered as the "most stable". The software automatically selected the most stable frame as the frame that showed the most similarities with preceding and succeeding frames. Then a large, central region-of-interest (ROI; one-fourth of the total shear-window size) on that frame was automatically drawn to exclude possible border artifacts. The SWE measurement corresponding to the mean stiffness value ( $E_{\text {mean }}$, expressed in $\mathrm{kPa}$ ) was extracted from the selected ROI.

To evaluate the quality of the SWE measurements, AAE software computed three quantitative indexes: i) the temporal variability (TV) estimated as the mean elasticity-value difference between adjacent video-clip frames, with the higher the TV corresponding to the larger the between-frame variability (hence TV $=0$ means that all frames were absolutely identical); ii) the percentage of pixel non-filling (PNF) on the elasticity map automatically measured in the frame AAE software identified as the most stable (the absence of shear-wave propagation yielding to uncolored pixels, values of $0 \%$ mean that all pixels are colored and $100 \%$ that none is colored); iii) the spatial variability (SV), computed as the standard deviation (SD) of the elasticity values in the most stable frame, with the higher the SV, the larger the spatial heterogeneity of the elasticity map (so $\mathrm{SV}=0$ means that the elasticity map is perfectly homogeneous). 
Other parameters. Also collected were body mass index (BMI), the skin-to-(right)-liversurface thickness (SLT) and the degree of liver heterogeneity. BMI was calculated from weight and height using the National Institutes of Health calculator, SLT was measured on the B-mode ultrasound, and heterogeneity was qualitatively assessed from B-mode images using a fourgrade scale (from $0=$ homogeneous, to $3=$ strongly heterogeneous).

Phantom study. SWE measurements were also made using an elastography phantom (model 059 CIRS, Norfolk, Virginia, USA) that was moved using a motion controller at speeds of 0.5 , 1, 2, 3, 4 and $6 \mathrm{~mm} / \mathrm{s}$. Before moving the phantom, four different $\mathrm{SWE}$ acquisitions were obtained for each speed after repositioning the transducer. For each speed, the phantom was moved from left to right, then from right to left, with this cycle being repeated three times. During each cycle, four frames were frozen and SWE measurements were made on them using the SWE-quantification Qbox system included in the Aixplorer. During each cycle, a circular ROI (6-mm diameter) was placed in the same homogeneous background area of the phantom and the mean and SD elasticity values were recorded.

Statistical Analyses. Bivariate adjustments and correlations between apnea and FB SWE values were computed for the whole population and different groups that were constituted based on clinical and morphological characteristics (liver homogeneity, BMI and SLT) and clip quality (TV, PNF and SV). Pearson's correlation coefficients $(r)$ and significance levels $(P)$ were determined for each category. Linear-regression analyses between mean SWE values obtained during apnea and FB were calculated for each group using an intercept value of 0 to establish the relationship between the two breathing modes and extract the slope $(y)$ corresponding to: $E_{\text {mean }}(\mathrm{FB})=y * E_{\text {mean }}$ (apnea). Because the clip-quality indexes had previously been defined 
for apnea, the TV, PNF and SV of the apnea images were used to classify the clip-quality subgroups. Concerning the phantom study, differences between the mean SWE values obtained without and with motion at different speeds were compared using Student's unpaired $t$-test. For the statistical analyses, patient and liver parameters were classified into categories: i) B-mode liver heterogeneity was classified as homogeneous (grade 0 or 1 ) or heterogeneous (grade 2 or 3); ii) BMI categories of patients were defined as normal-weight (BMI $\leq 25)$ or overweight patients (BMI >25; iii) SLT values were classified as normal (SLT <20 mm) or elevated (SLT $\geq 20 \mathrm{~mm}$ ).

The percentage of variation (PV) of each quality index $k$ (TV, PNF or SV) was computed as the normalized difference between the apnea (AP) and FB modes using the formula:

$P V(\%)=\left(k_{F B}-k_{A P}\right) / k_{A P}$

A positive index means degradation of the parameter in FB mode compared to apnea; a negative index indicates improvement.

\section{Results}

Clinical Study. Among the 109 patients, 10 were excluded because their SWE acquisitions failed to provide exploitable data in FB (4/109), apnea (2/109) or both breathing modes (4/109). The narrow intercostal space limiting the positioning of the probe and the absence of compliance in controlling breathing were the main reasons. Among the remaining 99 patients, 2 exams were excluded from the statistical analysis because measurements were aberrant (above 48 and $60 \mathrm{kPa}$ in apnea for values below $13 \mathrm{kPa}$ in FB). Hence, 97 patients (33 women and 64 men; mean \pm SD age $54 \pm 13$ years), i.e. 97 paired FB and apnea SWE measurements were analyzed. Patients' BMIs ranged from 16.5 to $44.9(25.4 \pm 4.9)$. 
Comparisons of FB and Apnea SWE Values. The mean SWE values of the entire study population (97 patients) obtained in $\mathrm{FB}$ was $9.5 \mathrm{kPa}$ (range 1.6 to $27.7 \mathrm{kPa}$ ), and was significantly lower than in apnea: $12.6 \mathrm{kPa}$ (range 2.0 to $60.7 \mathrm{kPa})(P<0.0001)$ (Fig. 1). The Pearson's correlation coefficient between FB and apnea elasticity values was $r=0.60$ $(P<0.0001)$. Table 1 reports the comparisons of mean SWE values obtained during FB and apnea, for the different liver, BMI and SLT categories. Corresponding scatter plots are provided in Fig. 2.

Comparisons of SWE Elasticity-Map Quality Indexes. Mean values of elasticity obtained during FB and apnea respectively, were 1.10 and $0.88 \mathrm{kPa}(P<0.005)$ for $\mathrm{TV}, 15.2$ and $8.2 \%(P<$ $0.0001)$ for PNF, and 5.0 and $4.8 \mathrm{kPa}(P=0.42)$ for SV. Comparisons of mean SWE values obtained during FB and apnea as a function of quality indexes are provided in Table 2. Table 3 shows the percentages of variation of quality-index (TV, PNF and SV) between apnea and FB modes for the different patient categories. Table 4 reports the two-tailed $t$-test $P$-values for the differences between the quality-index values only during apnea.

Phantom study. Mean SWE values between rest and the different motion speeds did not differ significantly (Fig. 3); left-to-right and right-to-left motions were combined because the motion direction did not influence the elasticity value.

\section{Discussion}

Ultrasound elastography was initially restricted to strain imaging, which estimated the tissue deformation resulting from compression by a static mechanical force. Obtaining those measurements in the liver required breath-holding throughout the acquisition of the pre- and 
post-compressive frames to limit artifacts due to breathing movements. The current consensus for SWE liver imaging also imposes breath-holding. However, because the technological principle of SWE is based on ultrafast imaging, we thought that the breath-holding constraint should be tested. Indeed, breath holding depends on the ability of the patient to control his respiration and it is well known in clinical practice that the quality of breath holding varies from a patient to another. Additionally, apnea might not be obtained in young children. In this regard, Franchi et al.'s recent results showed that SWE liver measurements in children under FB conditions were feasible and accurate [11].

In our study, FB- and apnea-stiffness values were correlated for the whole population and particularly for homogeneous livers and normal BMI and SLT categories. Correlations were weaker for unfavorable clinical conditions (high BMI and high SLT) and heterogeneous livers. Previous studies on liver SWE feasibility in breath holding conditions showed that the BMI might affect the rate of reliable measurements but does not influence the estimated elasticity values $[12,13]$. It thus seems that the weaker correlation between apnea and free breathing estimates for high BMI should be attributed to the difficulty to obtain reliable measurements when the BMI increases, independently of the breathing status.

Our study also showed that the mean liver-stiffness measurements under FB were significantly lower than those acquired under apnea. These differences were both observed under favorable and unfavorable clinical conditions. The decrease in liver stiffness measurements under breath holding when compared to those obtained during FB was of about 20 to $25 \%$.

These results suggest that, under favorable clinical conditions and when the liver is homogeneous, apnea is not needed, as long as the operator keeps in mind that SWE values under FB are lower than what would have been under apnea. However, under unfavorable clinical conditions or when the liver is heterogeneous, FB SWE measurements are not reliable. 
Two hypotheses can be put forward to explain why measured elasticity values are lower during FB than apnea. A first hypothesis would be that SWE values are wrongly estimated during FB because of inaccurate shear-wave-velocity estimation. Indeed, the instrument manufacturer recommends acquisition under apnea. Although shear-wave velocity is much faster than respiratory motion, it might be necessary to accumulate several consistent wave propagations to reconstruct the elasticity map. The setting parameters, especially persistence, are probably important to take into consideration. However, although motion can explain the poorer image quality, it does not seem to explain why FB values were consistently lower than those obtained during apnea. . A second hypothesis is that breath-holding could induce the equivalent of a Valsalva maneuver that would increase central venous pressure followed by an increase of the liver stiffness. Indeed, the influence of venous pressure on liver-stiffness measurements has been demonstrated in physiological and pathological conditions [14]. An experimental study on pigs [14] demonstrated that liver stiffness measures with transient elastography (Fibroscan, Echosens) was directly affected by central venous pressure, which is increased during a Valsalva maneuver. In addition, a recent study using SWE technology on 115 cirrhotic patients showed that liver-stiffness measurements were strongly correlated with the hepatic venous pressure gradient [15]. Hence, asking patients to hold their breath could lead to overestimation of liver stiffness.

Our phantom-study observations helped distinguish between those two hypotheses. Indeed, SWE values obtained with and without phantom motion were similar. Furthermore, the phantom's motion speed did not influence SWE values. Thus, we can hypothesize that discrepancies between stiffness values measured during FB and apnea in clinical practice originate from physiological changes in the liver between the two breathing modes that might be due to the elevated central venous pressure during apnea. 
Liver-stiffness measurements obtained during different breathing phases were described in previous papers where patients were asked to hold their breath [15-17]. The authors of those studies considered the same two breathing phases and found that liver stiffness at end-expiration was significantly higher than that at end-inspiration. The ESFUMB guidelines recommend transiently stopping breathing in a neutral position as it seems to be optimal for liver stiffness measurements[9]. In our study, the measurements were obtained under normal routine conditions. For apnea measurements, patients were just asked to block their respiration while in quiet breathing, without specific instructions as to how. Apnea could thus have occurred at the end of inspiration, at the end of expiration or at some time in-between. It is probable that for most patients, apnea occurred in a neutral position. This information could have been interesting to record and analyze, which is a limit of this work. Nonetheless, the study reproduces routine practice. Concerning Free Breathing measurements, they were obtained under conditions as close as possible to normal, quiet respiratory movements (including alternate inspiratory and expiratory cycles and apnea), which might vary from a patient.

The quality of the SWE-acquired data plays an important role in the accuracy and variability of the measurements. Inter or intra observer variabilities remain a critical question in SWE imaging in clinical practice. In the current study, the use of the AAE software allowed to limit the human factor bias because the SWE quantitative values were computed automatically in post processing from a 10 second clip that allowed the automatic selection of the most stable frame and ROI for stiffness measurements. The AAE automatic measurement is reliable as it was previously shown that the variability between the AAE measurement and the radiologist's measurement was similar to that obtained between two radiologists analyzing the same clip in post-processing (around 15\%) [10]. 
The originality of the current study is that the SWE elasticity-map quality was evaluated through quantitative parameters computed by the AAE software: TV ,PNF and SV. In our study, the quality of the FB clips was generally poorer than that of apnea videos, as expected. The factor the most strongly degraded was the degree of inbox filling which was represented by PNF ( $>85 \%$ degradation in FB vs. apnea). PNF degradation during FB was more pronounced for heterogeneous than homogeneous livers. Degradation of the inbox pixel filling from apnea to FB was worse for patients with $\mathrm{BMI} \leq 25$ and SLT $<20 \mathrm{~mm}$ (considered favorable clinical morphologies) than for the others. Nevertheless, PNF values during FB remained lower in the group with favorable clinical morphologies than the others. The SWE-video clips acquired during FB were also significantly more instable (increased TV values) when compared to those acquired during apnea $(P<0.005)$ especially in heterogeneous livers. Unlike other quality parameters, SV values were not significantly degraded by FB. In summary, our results showed that the liver echo texture plays an important role on the quality of the elasticity map during free breathing, with a more pronounced degradation of the PNF and TV parameters for heterogeneous livers rather than homogeneous. This confirms the expected finding that homogeneous tissues are much less influenced by motion than heterogeneous ones, as an echotexture with fluctuating features will lead to much more motion-induced blur than a plain texture. Liver echotexture evaluated on the Bmode image can be questionable as it is a subjective parameter which, to our knowledge, has not been previously reported in this field. In our study, in order to limit the bias, the four grade scale was gathered into two categories (homogeneous, heterogeneous). Contrarily to the heterogeneity factor, unfavorable bodyshapes did not worsen the SWE quality map from apnea to free breathing conditions. Our results suggest that the SWE map acquired during free breathing will provide measurements as reliable as in apnea when the liver is homogeneous. 
In summary, stiffness values estimated from FB and apnea acquisitions remain correlated especially for favorable clinical conditions of bodyshape and liver echo texture, but SWE values acquired during FB were consistently lower than those obtained during apnea. The phantom study indicated that the SWE-value discrepancies between breathing modes do not originate from the SWE technique and supported the hypothesis that hemodynamics changes induced by breathing conditions plays a role in the variation of liver stiffness in the same patient. The SWE examination under free breathing conditions is more comfortable for the patient in clinical practice and should provide acceptable SWE quality map for reliable measurements in some clinical conditions while keeping in mind that estimated SWE values will be lower than those that would be obtained during apnea. Comparative studies of liver stiffness, e.g. for patient follow-up, definitely need to take the breathing mode into account.

\section{References}

[1] G.S. Gherlan, Liver ultrasound elastography: More than staging the disease, World J Hepatol. 7 (2015) 1595-1600.

[2] T. Shiina, K.R. Nightingale, M.L. Palmeri, T.J. Hall, J.C. Bamber, R.G. Barr, L. Castera, B.I. Choi, Y.-H. Chou, D. Cosgrove, C.F. Dietrich, H. Ding, D. Amy, A. Farrokh, G. Ferraioli, C. Filice, M. Friedrich-Rust, K. Nakashima, F. Schafer, I. Sporea, S. Suzuki, S. Wilson, M. Kudo, WFUMB Guidelines and Recommendations for Clinical Use of Ultrasound Elastography: Part 1: Basic Principles and Terminology, Ultrasound in Medicine \& Biology. 41 (2015) 1126-1147.

[3] B.S. Garra, Elastography: history, principles, and technique comparison, Abdom Imaging. 40 (2015) 680-697.

[4] T. Deffieux, J.-L. Gennisson, L. Bousquet, M. Corouge, S. Cosconea, D. Amroun, S. Tripon, B. Terris, V. Mallet, P. Sogni, M. Tanter, S. Pol, Investigating liver stiffness and viscosity for fibrosis, steatosis and activity staging using shear wave elastography, J. Hepatol. 62 (2015) 317-324.

[5] Y. Huang, G.-J. Liu, B. Liao, G.-L. Huang, J.-Y. Liang, L.-Y. Zhou, F. Wang, W. Li, X.Y. Xie, W. Wang, M.-D. Lu, Impact factors and the optimal parameter of acoustic structure quantification in the assessment of liver fibrosis, Ultrasound Med Biol. 41 (2015) 2360-2367.

[6] H.-J. Kim, H.-K. Lee, J.-H. Cho, H.-J. Yang, Quantitative comparison of transient elastography (TE), shear wave elastography (SWE) and liver biopsy results of patients with chronic liver disease, J Phys Ther Sci. 27 (2015) 2465-2468. 
[7] N. Yada, T. Sakurai, T. Minami, T. Arizumi, M. Takita, S. Hagiwara, K. Ueshima, H. Ida, N. Nishida, M. Kudo, A Newly Developed Shear Wave Elastography Modality: With a Unique Reliability Index, Oncology. 89 Suppl 2 (2015) 53-59.

[8] L. Sandrin, M. Tanter, S. Catheline, M. Fink, Shear modulus imaging with 2-D transient elastography, IEEE Trans Ultrason Ferroelectr Freq Control. 49 (2002) 426-435.

[9] D. Cosgrove, F. Piscaglia, J. Bamber, J. Bojunga, J.-M. Correas, O.H. Gilja, A.S. Klauser, I. Sporea, F. Calliada, V. Cantisani, M. D’Onofrio, E.E. Drakonaki, M. Fink, M. FriedrichRust, J. Fromageau, R.F. Havre, C. Jenssen, R. Ohlinger, A. Săftoiu, F. Schaefer, C.F. Dietrich, EFSUMB, EFSUMB guidelines and recommendations on the clinical use of ultrasound elastography. Part 2: Clinical applications, Ultraschall Med. 34 (2013) 238253.

[10] C. Pellot-Barakat, M. Lefort, L. Chami, M. Labit, F. Frouin, O. Lucidarme, Automatic assessment of shear wave elastography quality and measurement reliability in the liver, Ultrasound Med Biol. 41 (2015) 936-943.

[11] S. Franchi-Abella, L. Corno, E. Gonzales, G. Antoni, M. Fabre, B. Ducot, D. Pariente, J.L. Gennisson, M. Tanter, J.-M. Corréas, Feasibility and Diagnostic Accuracy of Supersonic Shear-Wave Elastography for the Assessment of Liver Stiffness and Liver Fibrosis in Children: A Pilot Study of 96 Patients, Radiology. 278 (2016) 554-562.

[12] Z. Huang, J. Zheng, J. Zeng, X. Wang, T. Wu, R. Zheng, Normal liver stiffness in healthy adults assessed by real-time shear wave elastography and factors that influence this method, Ultrasound Med Biol. 40 (2014) 2549-2555.

[13] I. Sporea, R. Sirli, R. Mare, A. Popescu, S.C. Ivascu, Feasibility of Transient Elastography with $\mathrm{M}$ and XL probes in real life, Med Ultrason. 18 (2016) 7-10.

[14] G. Millonig, S. Friedrich, S. Adolf, H. Fonouni, M. Golriz, A. Mehrabi, P. Stiefel, G. Pöschl, M.W. Büchler, H.K. Seitz, S. Mueller, Liver stiffness is directly influenced by central venous pressure, J. Hepatol. 52 (2010) 206-210.

[15] T.Y. Kim, W.K. Jeong, J.H. Sohn, J. Kim, M.Y. Kim, Y. Kim, Evaluation of portal hypertension by real-time shear wave elastography in cirrhotic patients, Liver Int. 35 (2015) 2416-2424.

[16] M.H. Yun, Y.S. Seo, H.S. Kang, K.G. Lee, J.H. Kim, H. An, H.J. Yim, B. Keum, Y.T. Jeen, H.S. Lee, H.J. Chun, S.H. Um, C.D. Kim, H.S. Ryu, The effect of the respiratory cycle on liver stiffness values as measured by transient elastography, J. Viral Hepat. 18 (2011) 631-636.

[17] W. Ling, Q. Lu, J. Quan, L. Ma, Y. Luo, Assessment of impact factors on shear wave based liver stiffness measurement, Eur J Radiol. 82 (2013) 335-341. 


\section{Tables}

Table 1. Comparisons between Mean SWE Values Obtained during Free-Breathing (FB) or Apnea (AP) for Different Patient Categories.

\begin{tabular}{|c|c|c|c|c|c|c|c|}
\hline \multirow[b]{2}{*}{ Parameter } & \multirow[b]{2}{*}{ All } & \multicolumn{2}{|c|}{ Liver } & \multicolumn{2}{|c|}{ Body Mass Index } & \multicolumn{2}{|c|}{ Skin-to-Liver Thickness } \\
\hline & & Hom & Het & $\leq 25$ & $>25$ & $<20 \mathrm{~mm}$ & $\geq 20 \mathrm{~mm}$ \\
\hline Number & 97 & 64 & 33 & 55 & 42 & 74 & 23 \\
\hline FB & 9.5 & 8.9 & 10.7 & 8.1 & 11.3 & 8.6 & 12.3 \\
\hline$A P$ & 12.6 & 10.5 & 16.8 & 10.5 & 14.1 & 11.4 & 16.3 \\
\hline Pearson's $r$ & 0.48 & 0.76 & 0.37 & 0.68 & 0.48 & 0.67 & 0.39 \\
\hline$P$-value & $<0.00001$ & $<0.00001$ & 0.041 & $<0.00001$ & $<0.001$ & $<0.00001$ & 0.06 \\
\hline$y$ (slope) & 0.61 & 0.74 & 0.80 & 0.67 & 0.77 & 0.72 & 0.74 \\
\hline
\end{tabular}

Liver: Homogeneous (Hom), Heterogeneous (Het); Body mass index: normal weight ( $\leq 25)$, overweight $(>25)$; skin-to-liver thickness: thin ( $<20 \mathrm{~mm})$, thick ( $\geq 20 \mathrm{~mm})$.

Table 2. Comparisons between Mean SWE Stiffness Values Obtained during Free-Breathing (FB) or Apnea (AP) for the Different Quality-Indexes.

\begin{tabular}{lcccccc}
\hline & \multicolumn{2}{c}{ Temporal Variability } & \multicolumn{2}{c}{ \% Non-Filled Pixels } & \multicolumn{2}{c}{ Spatial Variability } \\
\hline Parameter & $\leq 1$ & $>1$ & $\leq 10 \%$ & $>10 \%$ & $\leq 4 \mathbf{k P a}$ & $>4 \mathbf{k P a}$ \\
\hline Number & 66 & 31 & 76 & 21 & 49 & 48 \\
FB & 9.2 & 9.9 & 9.3 & 9.9 & 7.8 & 11.2 \\
AP & 11.7 & 12.0 & 11.9 & 11.1 & 8.6 & 15.0 \\
Pearson's $r$ & 0.57 & 0.67 & 0.70 & 0.30 & 0.80 & 0.36 \\
$P$-value & $<0.00001$ & $<0.0001$ & $<0.00001$ & 0.186 & $<0.00001$ & 0.011 \\
$Y$ (slope) & 0.73 & 0.75 & 0.73 & 0.78 & 0.86 & 0.69 \\
\hline
\end{tabular}


Table 3. Comparisons between Free-Breathing (FB) and Apnea (AP) Mean values with P-values and percentage of variation (PV) for the Three Quality Indexes and Seven Patient Categories.

\begin{tabular}{|c|c|c|c|c|c|c|c|c|}
\hline & & & & & Body & Index & Skin-to-Li & hickness \\
\hline Index & Parameters & All & Hom & Het & $\leq 25$ & $>25$ & $<20 \mathrm{~mm}$ & $\geq 20 \mathrm{~mm}$ \\
\hline 구 & Mean FB & 1.10 & 1.01 & 1.27 & 1.09 & 1.10 & 1.07 & 1.18 \\
\hline 은 & Mean AP & 0.88 & 0.92 & 0.81 & 0.85 & 0.92 & 0.83 & 1.03 \\
\hline$\overline{\bar{N}}$ & $P$-value & $<0.005$ & 0.2989 & $<0.001$ & $<0.05$ & 0.0820 & $<0.01$ & 0.3522 \\
\hline$\stackrel{E}{\varrho}$ & PV & 25 & 10 & 57 & 28 & 20 & 29 & 15 \\
\hline & Mean FB & 15.24 & 13.47 & 18.80 & 13.93 & 16.88 & 14.85 & 16.41 \\
\hline$\frac{x}{0}$ & Mean AP & 8.17 & 7.80 & 8.89 & 6.88 & 9.78 & 5.93 & 14.79 \\
\hline 衰 & $P$-value & $<0.0001$ & $<0.005$ & $<0.05$ & $<0.005$ & $<0.05$ & $<0.0001$ & 0.5665 \\
\hline 2 & PV & 87 & 73 & 111 & 102 & 73 & 150 & 11 \\
\hline & Mean FB & 4.96 & 4.41 & 5.55 & 3.76 & 6.46 & 4.10 & 7.50 \\
\hline 可 & Mean AP & 4.77 & 4.25 & 5.80 & 3.47 & 6.38 & 3.89 & 7.35 \\
\hline$\frac{\pi}{3}$ & $P$-value & 0.423 & 0.621 & 0.443 & 0.286 & 0.854 & 0.384 & 0.822 \\
\hline के & PV & 4 & 4 & -4 & 8 & 1 & 5 & 2 \\
\hline
\end{tabular}

Liver: Homogeneous (Hom), Heterogeneous (Het); Body mass index: normal weight ( $\leq 25)$, overweight (>25); skin-to-liver thickness: thin $(<20 \mathrm{~mm})$, thick $(\geq 20 \mathrm{~mm})$.

Table 4. Differences Between the Quality-Index Values Obtained during Apnea for the Three Patient Categories.

\begin{tabular}{lccc}
\hline $\begin{array}{c}\text { Liver } \\
\text { Index }\end{array}$ & $\begin{array}{c}\text { Body Mass Index } \\
\mathbf{5} \mathbf{2 5} \mathbf{~ v s . ~} \mathbf{2 5}\end{array}$ & $\begin{array}{c}\text { Skin-to-Liver Thickness } \\
<\mathbf{2 0} \mathbf{~ m m ~ v s . ~} \mathbf{2 0} \mathbf{~ m m}\end{array}$ \\
\hline Temporal variability & 0.313 & 0.502 & 0.091 \\
\% Non-filled pixels & 0.689 & 0.257 & $<0.005$ \\
Spatial variability & $<0.05$ & $<0.0001$ & $<0.0001$ \\
Values are the $P$-values computed with Student's $t$-test.
\end{tabular}




\section{Figure Legends}

Fig. 1. Mean SWE stiffness values $(\mathrm{kPa})$ during free-breathing vs. apnea for the whole population $(\mathrm{n}=97)$. The linear relationship between the two states is $y=0.7375 x$ with $r^{2}=$ 0.363 .

Fig. 2. Mean SWE stiffness values ( $\mathrm{kPa}$ ) obtained during free-breathing versus apnea for $\mathrm{A}$ ) homogeneous $(O)$ and heterogeneous $(x)$ livers, $B$ ) normal $(O)$ and high body mass indexes $(\mathrm{BMI})(\times), \mathrm{C})$ normal $(\bigcirc)$ and large skin-to-liver-surface thickness (SLT) $(\times)$.

Fig. 3. Mean and SD SWE stiffness values $(\mathrm{kPa})$ in a homogeneous region of the phantom for different motion speeds. 


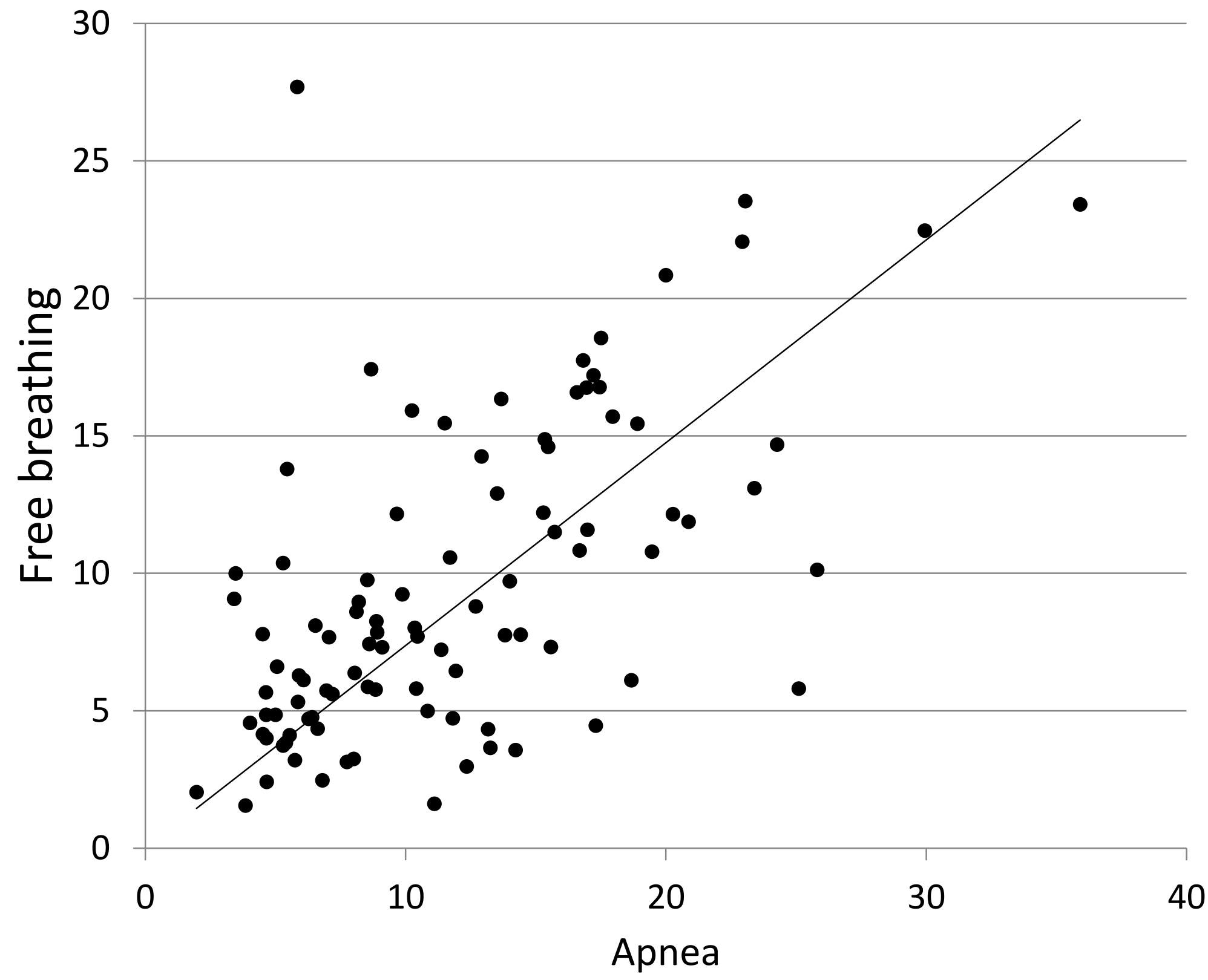

Fig 1 


\section{A}

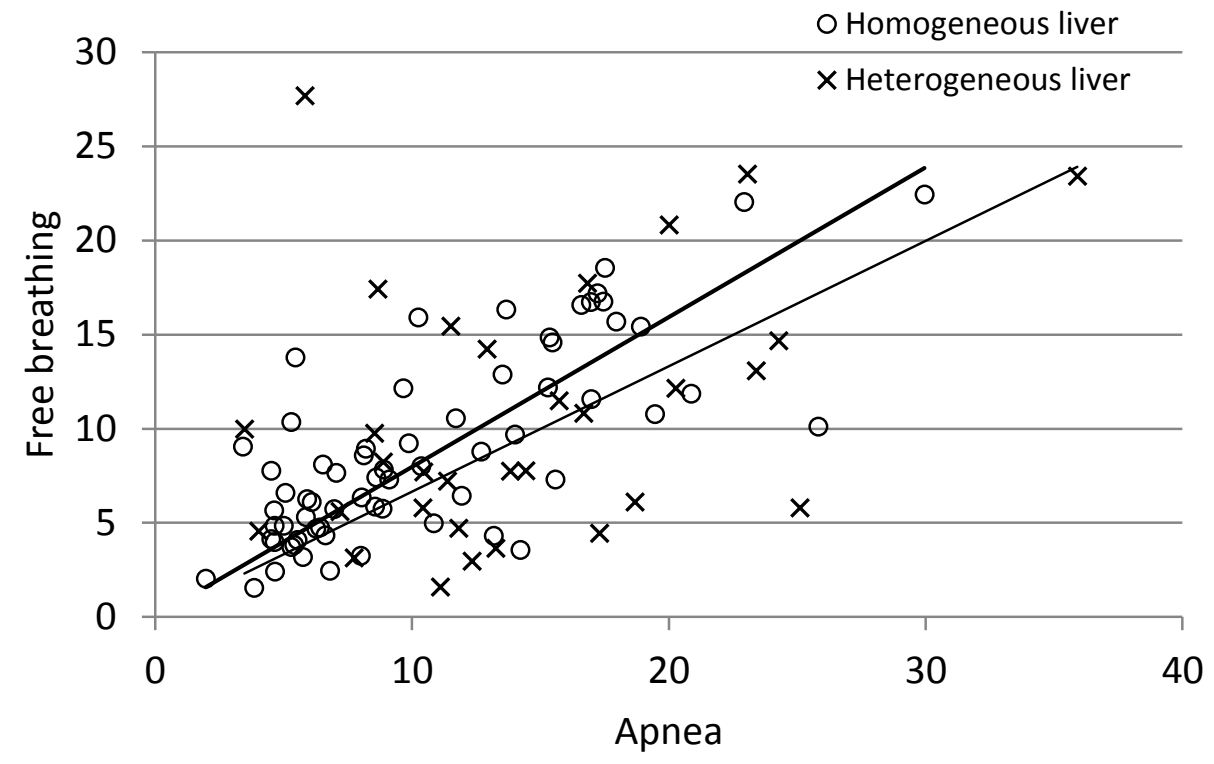

B

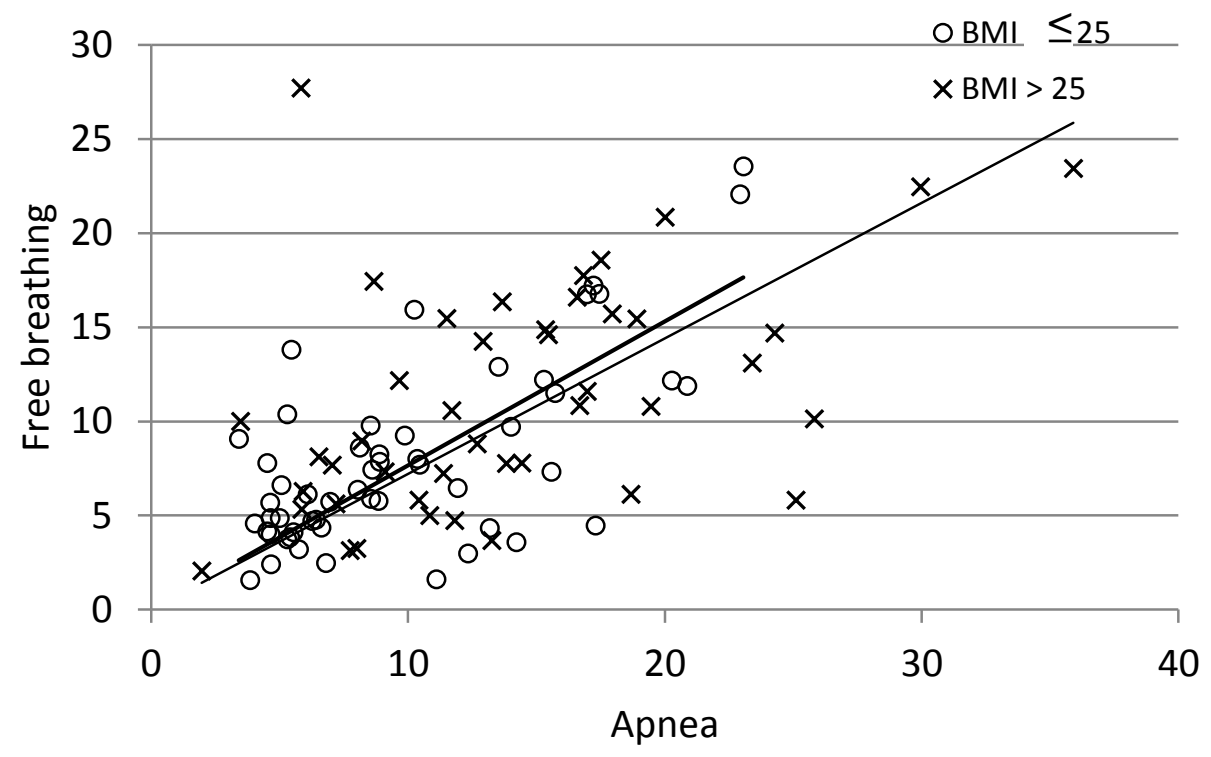

C

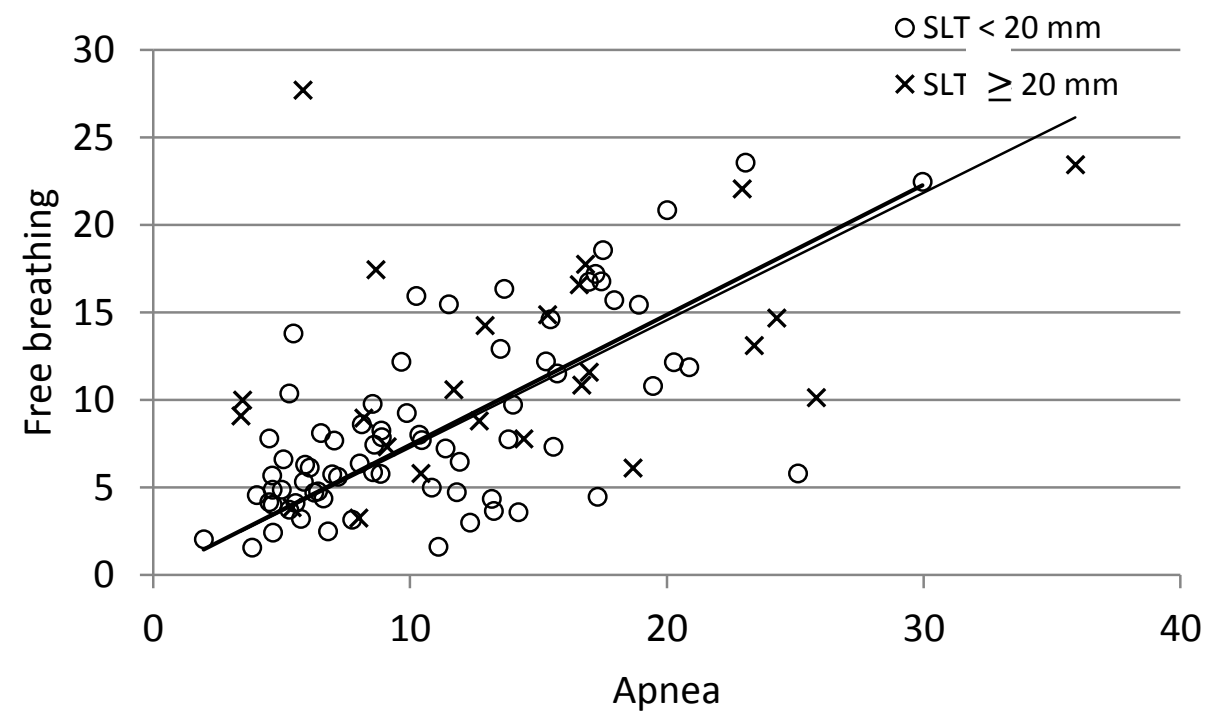




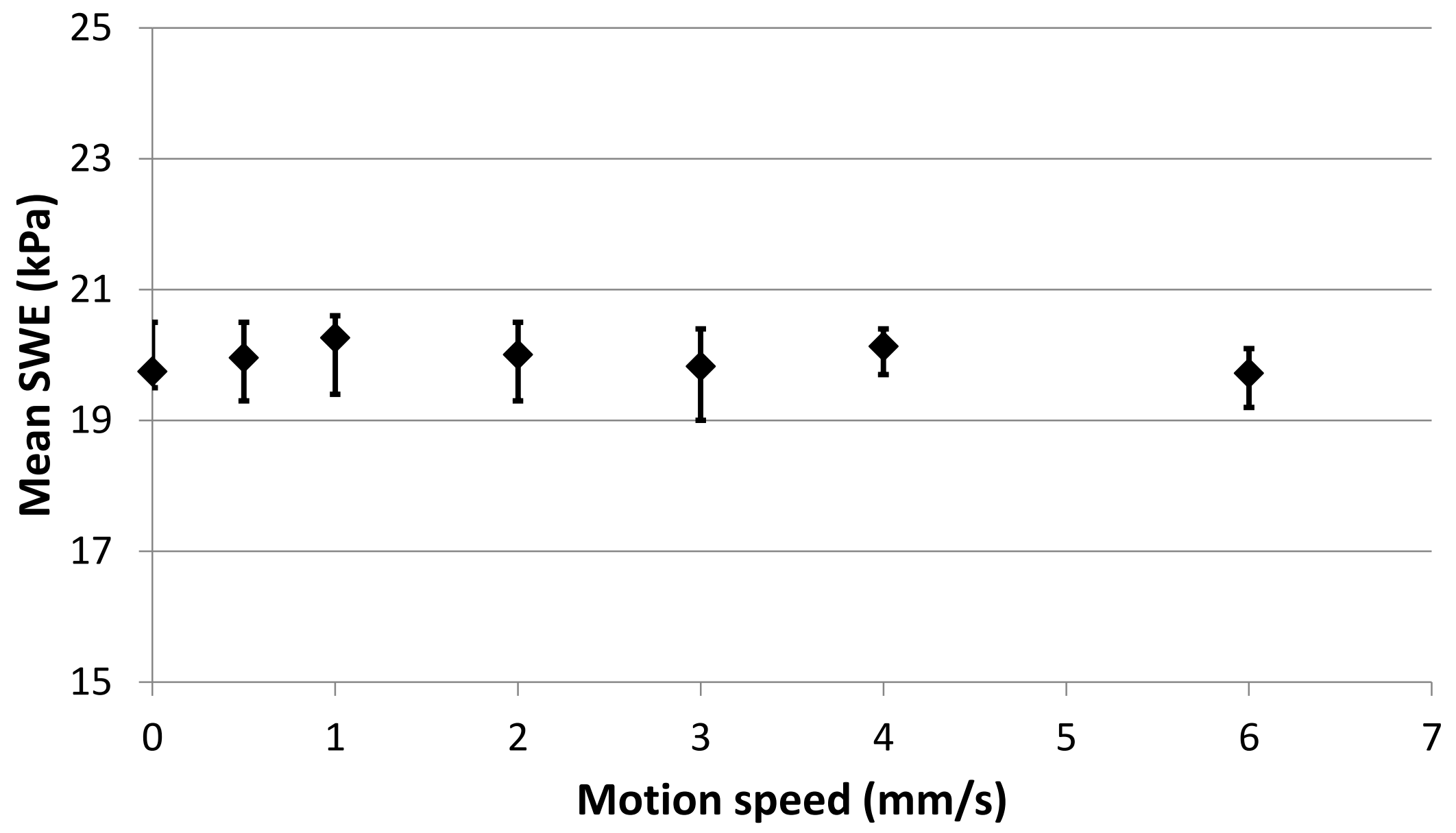

